Check for updates

Cite this: Mater. Adv., 2021, 2, 2031

Received 27th January 2021, Accepted 19th February 2021

DOI: $10.1039 / \mathrm{d} 1 \mathrm{ma} 00068 \mathrm{c}$

rsc.li/materials-advances

\section{The performance of conjugated polymers as emitters for triplet-triplet annihilation upconversion $\dagger$}

\author{
Riley O'Shea, ${ }^{\mathrm{ab}}$ Can Gao, (D) ${ }^{\mathrm{c}}$ Tze Cin Owyong, $^{\mathrm{ab}}$ Jonathan M. White (D) $^{\mathrm{b}}$ and \\ Wallace W. H. Wong iD *ab
}

\begin{abstract}
A series of poly(phenylene-ethynylene) copolymers with various aryl spacer units were synthesized for use as emitters in triplet-triplet annihilation upconversion. The upconversion performance of these conjugated polymers was compared to that of well-known poly(phenylene-vinylene) polymers, MEH-PPV and super yellow, in chloroform solution. The copolymer containing anthracene units outperformed both reference polymers recording a maximum upconversion quantum yield of $0.18 \%$.
\end{abstract}

\section{Introduction}

Triplet-triplet annihilation upconversion (TTA-UC), also known as triplet fusion upconversion, is a photochemical process by which two lower energy photons can be combined to produce one photon of higher energy. ${ }^{1}$ It sees use in raising the efficiency of solar cells above their thermodynamic limit (the Shockley-Queisser limit). ${ }^{2,3}$

Two chromophores are required in a typical TTA-UC system a triplet sensitizer and an annihilator/emitter. ${ }^{1}$ The triplet sensitizer absorbs a photon promoting it to its singlet excited state (Fig. 1a). Intersystem crossing (ISC) leads to the formation of the triplet excited state on the sensitizer. This triplet exciton can then be transferred to an emitter molecule via triplet energy transfer (TET). As the triplet exciton population of emitters builds, two triplet excitons can combine leading to triplet-triplet annihilation (TTA) generating a higher energy singlet exciton. This singlet exciton then undergoes radiative decay releasing a photon that is higher in energy than the photon absorbed by the sensitizer.

Transition metal complexes are most commonly used as triplet sensitizers but metal chalcogenide quantum dots have also been used. $^{4,5}$ As for the emitter component, polycyclic aromatic hydrocarbon molecules have been widely investigated. ${ }^{6-9}$ Much less

\footnotetext{
${ }^{a}$ ARC Centre of Excellence in Exciton Science, School of Chemistry, University of Melbourne, Parkville, VIC 3010, Australia. E-mail: wwhwong@unimelb.edu.au

${ }^{b}$ School of Chemistry, Bio21 Institute, University of Melbourne, Parkville, VIC 3010, Australia

${ }^{c}$ Beijing National Laboratory for Molecular Sciences, Key Laboratory of Organic Solids, Institute of Chemistry, Chinese Academy of Sciences, Beijing, China

$\dagger$ Electronic supplementary information (ESI) available. CCDC 1992339. For ESI and crystallographic data in CIF or other electronic format see DOI: 10.1039/ d1ma00068c
}

well-established is the use of conjugated polymers as emitters in TTA-UC. ${ }^{10-14}$ It was shown in theoretical models that the extended conjugation of these materials leads to improved triplet exciton diffusion which may assist in TTA-UC performance. ${ }^{15}$ Measurements of TTA-UC systems containing conjugated polymers showed some promise but accurate comparison of TTA-UC efficiency has not been reported. ${ }^{14,16}$ In this study, the TTA-UC

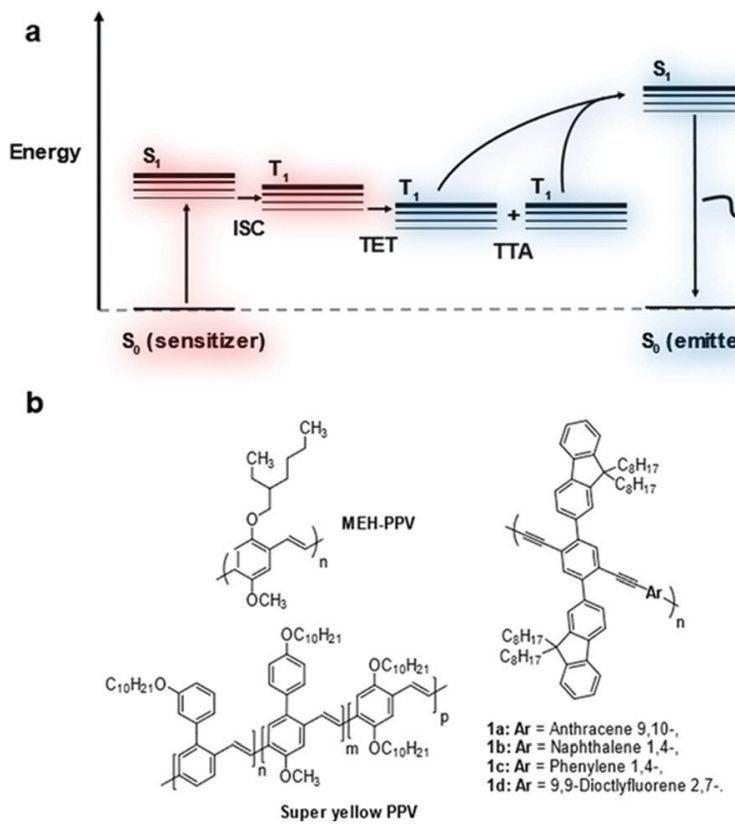

Fig. 1 Jablonski diagram depicting the process involved for TTA-UC (a). Structures of poly(phenylene-vinylene)s, MEH-PPV and super yellow, and copolymers based on poly(phenylene-ethynylene) investigated as emitters for TTA-UC (b) 
performance of several conjugated polymers in solution was investigated and compared (Fig. 1b).

There are some important parameters to consider when measuring TTA-UC performance. ${ }^{17}$ The TTA-UC efficiency is measured by the upconversion quantum yield $\left(\Phi_{\mathrm{UC}}\right)$, which is a product of all the quantum yields for photochemical processes involved, given by the equation:

$$
\Phi_{\mathrm{UC}}=\Phi_{\mathrm{ISC}} \Phi_{\mathrm{TET}} \Phi_{\mathrm{TTA}} \Phi_{\mathrm{PL}}
$$

where $\Phi_{\text {ISC }}$ is the intersystem crossing quantum yield, $\Phi_{\text {TET }}$ is the triplet energy transfer quantum yield, $\Phi_{\text {TTA }}$ is the TTA quantum yield, and $\Phi_{\mathrm{PL}}$ is the photoluminescent quantum yield. ${ }^{16} \Phi_{\text {TTA }}$ can be further separated into the contact triplet pair formation efficiency and the factor $f{ }^{16}$ which is a measure of the probability of generating the singlet excited state after TTA, since singlet, triplet and quintet states are all possible outcomes. This factor may differ significantly between different emitter molecules. ${ }^{18-20}$

In measuring $\Phi_{\mathrm{UC}}$, a relative measurement is typically preferred due to its simplicity. ${ }^{21}$ This can be done by comparing the integrated photoluminescence intensity of a standard fluorescent dye with a known $\Phi_{\mathrm{PL}}$ and that of the upconversion dye pair, eqn (2):

$$
\Phi_{\mathrm{UC}}=\Phi_{\mathrm{std}}\left(\frac{A_{\mathrm{std}}}{A_{\mathrm{Unk}}}\right)\left(\frac{I_{\mathrm{Unk}}}{I_{\mathrm{std}}}\right)\left(\frac{\eta_{\mathrm{Unk}}}{\eta_{\mathrm{std}}}\right)^{2}
$$

where $A$ is the absorbance, $I$ is the integrated photoluminescence intensity, $\eta$ is the refractive index of the medium and $\Phi_{\text {std }}$ is the $\Phi_{\mathrm{PL}}$ of the standard. The $\Phi_{\mathrm{UC}}$ value of upconversion systems can also be determined using absolute quantum yield method. ${ }^{22}$ Previous studies on conjugated polymers in TTA-UC did not report on the $\Phi_{\mathrm{UC}}$ value determined using either absolute or relative method. ${ }^{10-14}$

Herein, the $\Phi_{\mathrm{UC}}$ of solutions containing commercially available poly(phenylene-vinylene)s, MEH-PPV and super yellow, were measured using an absolute quantum yield method with mesotetraphenyl-tetrabenzoporphine palladium complex (PdTPTBP) as the triplet sensitizer. We then compare the performance of $\mathrm{MEH}$ PPV and super yellow to a series of poly(phenylene-ethynylene) copolymers. The TTA-UC samples were in a similar concentration range in chloroform solution and were optimized for maximum TTA-UC emission.

\section{Results and discussion}

A series of copolymers based on poly(phenylene-ethynylene) (PPE) was devised bearing dioctylfluorene sidechains to increase polymer solubility and to reduce molecular interactions that increase the rate of non-radiative decay (Fig. 1). It is well-known that PPEs with alkyl sidechains can show strong molecular association leading to low photoluminescence quantum yield. ${ }^{23}$ The aryl comonomer unit was varied to fine tune the photophysical properties of the polymer backbone.

The bis-ethyne monomer $\mathbf{4}$ was synthesized by Suzuki coupling followed by treatment with TBAF to remove the TMS protecting
Table 1 Properties of PPE copolymers 1a-d

\begin{tabular}{lcrrrrr}
\hline & $M_{\mathrm{w}}\left(\mathrm{g} \mathrm{mol}^{-1}\right)$ & $D$ & $\mathrm{DP}^{a}$ & $\mathrm{Abs} \lambda_{\max }(\mathrm{nm})$ & $\mathrm{PL} \lambda_{\max }(\mathrm{nm})$ & $\Phi_{\mathrm{PL}}^{b}(\%)$ \\
\hline 1a & 11600 & 2.0 & 11 & 321 & 533 & $49.5 \pm 0.2$ \\
1b & 5310 & 1.6 & 7 & 319 & 462 & $97.8 \pm 2.1$ \\
1c & 7070 & 1.6 & 9 & 320 & 450 & $74.5 \pm 1.5$ \\
1d & 5910 & 1.9 & 6 & 323 & 445 & $93.8 \pm 2.2$
\end{tabular}

${ }^{a} \mathrm{DP}=$ degree of polymerization. ${ }^{b}$ Solution $\Phi_{\mathrm{PL}}$ measured at $10 \mu \mathrm{g} \mathrm{mL}{ }^{-1}$ concentration of polymer in chloroform using anthracene as a reference with excitation at $350 \mathrm{~nm}$.

groups in $74 \%$ yield (Fig. S2, ESI $\dagger$ ). The single crystal structure of monomer 4 was obtained by slow evaporation from a chloroform solution. The structure showed a slip stacked packing, with a symmetrical kink in of one of the octyl chains on each of the fluorene units caused by close packing (for further details see Table S1, ESI $\dagger$ ). The para-aryldibromide monomers 5a-d were either commercially available or synthesized using literature methods (see ESI $\dagger$ for details).

The polymers were synthesized using Sonogashira polycondensation between monomer $\mathbf{4}$ and dibromide monomers 5a-d, with reaction yields varying between $41-66 \%$ (Table S2, ESI $\dagger$ ). The molecular weight of the polymers was found to be fairly low $<15000 \mathrm{~g} \mathrm{~mol}^{-1}$ (Table 1, see ESI, $\dagger$ Fig. S7 for GPC traces). Attempts to increase these values proved to be unsuccessful. Neither longer reaction times nor higher temperatures had any effect on the molecular weight. Similarly, no change was noticed when the corresponding para-aryldiiodides were used in place of the para-aryldibromides 5 . When using $\mathrm{Pd}(\mathrm{dppf}) \mathrm{Cl}_{2}$ as the catalyst, a slightly higher molecular weight range was obtained, however the polymers produced were weakly emissive. Similar PPEs reported in prior literature also exhibited low molecular weights with Sonogashira polycondensations. ${ }^{24-26}$ Higher molecular weights in homopolymers could be obtained by alkyne metathesis, ${ }^{27}$ however this method is difficult to implement for construction of copolymers. Concerning the polymer properties for TTA-UC, it should be noted that as little as 5 repeat units have been shown to be sufficient for similar photophysical properties as the corresponding PPE. ${ }^{24}$

The photoluminescence spectrum of the copolymers showed some variation in the peak emission with the most significant difference for anthracene copolymer 1a. The extended $\pi$ system of the anthracene in 1a led to the most red-shifted UV-Vis absorption edge and photoluminescence (PL) peak maxima in the series (Fig. 2). All copolymers have an absorption maximum at $\sim 320 \mathrm{~nm}$ which can be attributed to the fluorene sidechains. ${ }^{28}$ Copolymer $1 \mathrm{a}$ also showed vibronic features in its absorption and PL spectra reminiscent of the anthracene building block. The $\Phi_{\mathrm{PL}}$ of the polymers ranged from $50 \%$ to $100 \%$ (Table 1), with the anthracene derivative 1a being the least emissive and the naphthalene derivative $\mathbf{1 b}$ being the most emissive. The most likely reason for the $\Phi_{\mathrm{PL}}$ variations is the positioning of the fluorene sidechains and their effect on polymer-polymer interactions. Some insights are discussed in the DFT calculations section below. These PLQY values are reminiscent of many PPE copolymers, with solution $\Phi_{\mathrm{PL}}$ values 

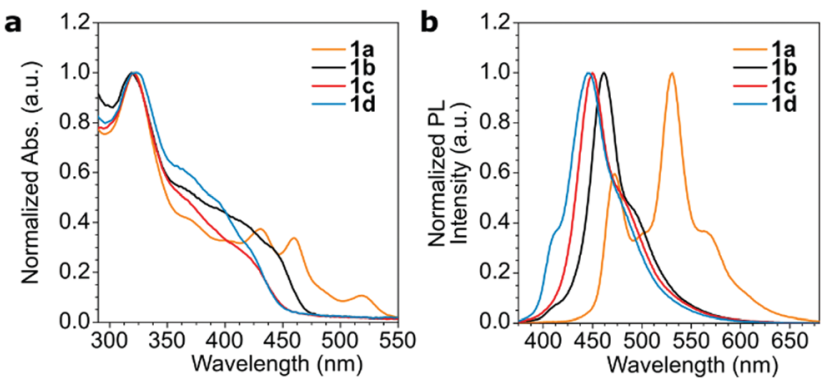

Fig. 2 Normalized UV-Vis absorption (a) and photoluminescence (b) spectra of copolymers 1a-d in chloroform solution. Excitation wavelength: $350 \mathrm{~nm}$.

typically being in excess of $50 \%$, with many of those containing simple alkyl side chains displaying solution $\Phi_{\mathrm{PL}}$ close to $100 \% .^{23,29}$

Next, the TTA-UC performance of the polymers were tested with PdTPTBP as the triplet sensitizer. The polymers $\left(0.25 \mathrm{mg} \mathrm{mL}^{-1}\right)$ and the PdTPTBP $(7.5 \mu \mathrm{M})$ were well-dissolved in chloroform as it was apparent from the absorption spectrum of the samples (Fig. S9, ESI $\dagger$ ). A red-to-green upconversion was observed with the anthracene-based PPE 1a with $632 \mathrm{~nm}$ excitation (Fig. 3). The upconversion emission intensity was optimized and found to occur using a polymer concentration of $0.25 \mathrm{mg} \mathrm{mL}^{-1}$ with a $7.5 \mu \mathrm{M}$ concentration of PdTPTBP. Surprisingly, none of the other PPE derivatives showed any upconversion emission under the same conditions. In fact, the polymers $\mathbf{1 b}-\mathbf{d}$ failed to quench the phosphorescence of PdTPTBP at $800 \mathrm{~nm}$ (Fig. 3). This is a clear indication that only $\mathbf{1 a}$ has appropriate triplet excited state energy to interact with the PdTPTBP sensitizer.

The upconverted emission maximum for the sample containing 1a was close to that of the conventional photoluminescence maximum for 1a at $540 \mathrm{~nm}$ but the emission profiles were rather different especially with the $600 \mathrm{~nm}$ banc pass filter used on the upconverted emission measurement (Fig. S15, ESI $\dagger$ ).

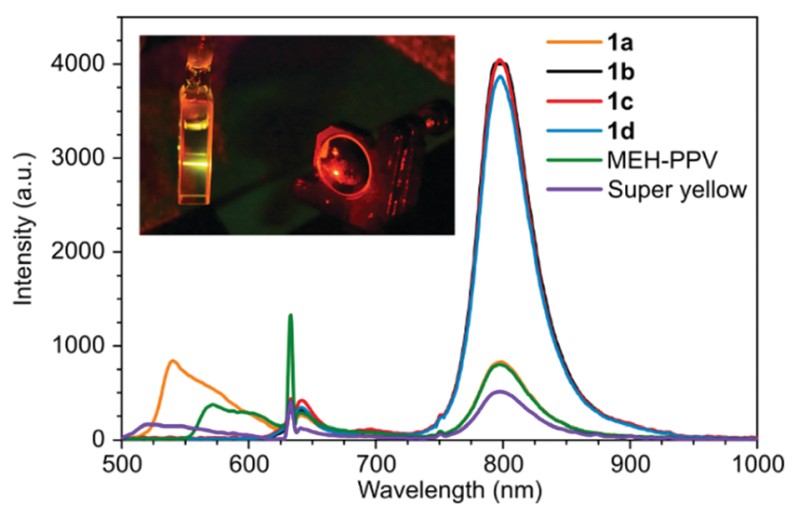

Fig. 3 Emission spectrum of samples containing copolymers $1 \mathbf{a}-\mathbf{d}$ $\left(0.25 \mathrm{mg} \mathrm{mL}^{-1}\right)$, MEH-PPV $\left(0.5 \mathrm{mg} \mathrm{mL}^{-1}\right)$, super yellow PPV $\left(0.5 \mathrm{mg} \mathrm{mL}^{-1}\right)$ with PdTPTBP $(7.5 \mu \mathrm{M})$ as the sensitizer in chloroform solution under $632 \mathrm{~nm}$ excitation. Phosphorescence of PdTPTBP was observed at $800 \mathrm{~nm}$ and the upconverted emission was observed at $500-600 \mathrm{~nm}$. The insert is a photo of a cuvette containing copolymer $1 \mathrm{a}$ and PdTPTBP showing yellow TTA-UC emission.
These copolymers were then compared to commercially available PPV emitters - MEH-PPV and super yellow - whose $\Phi_{\mathrm{UC}}$ was determined via an integrating sphere method under nitrogen atmosphere to be $0.039 \%$ and $0.029 \%$ respectively. These values were determined in chloroform solution with an

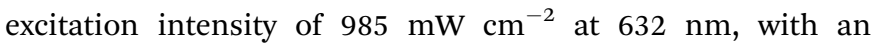
optimized polymer concentration of $0.5 \mathrm{mg} \mathrm{mL}^{-1}$ and РdTPTBP at $7.5 \mu \mathrm{M}$. Despite the higher $\Phi_{\mathrm{PL}}$ of super yellow at $69 \%$ compared to $27 \%$ for MEH-PPV, its $\Phi_{\mathrm{UC}}$ is lower than $\mathrm{MEH}-$ PPV. ${ }^{30,31}$ The lower upconversion intensity of super yellow and its substantially higher cost compared to MEH-PPV, makes MEH-PPV preferable as an upconversion standard. These commercial polymers serve as adequate standards, with a large degree of spectral overlap due to their broad photoluminescent emission in the same region displayed by the PPE copolymers.

The $\Phi_{\mathrm{UC}}$ values were measured as a function of the excitation intensity for 1a and MEH-PPV. A maximum $\Phi_{\mathrm{UC}}$ value of $0.18 \%$ was reached at $8910 \mathrm{~mW} \mathrm{~cm}^{-2}$ (Fig. 4). The $\Phi_{\mathrm{UC}}$ of $\mathrm{MEH}^{-}$ PPV reached a maximum value of $0.065 \%$ at $10500 \mathrm{~mW} \mathrm{~cm}^{-2}$. These values were also measured relative to super yellow PPV and found to have little to no relative error (Table S3, ESI $\dagger$ ). Although these are low values of $\Phi_{\mathrm{UC}}$ compared to the best solution TTA-UC systems, ${ }^{32}$ this work showed PPE materials are possible emitters and performed significantly better than both MEH-PPV and super yellow.

To gain insight into the effect of various aryl monomer building blocks on the properties of the polymers, density functional theory (DFT) calculations were performed (Fig. 5 and 6). A semi-empirical method (PM6) was used to optimize the geometry of these polymers, then the TD-DFT was performed with B3LYP/6-311G(d,p) to obtain the singlet and triplet excited state energies. As mentioned previously, the difference in the $\Phi_{\mathrm{PL}}$ values (Table 1) could be related to the positioning of the fluorene sidechains. For the naphthalene and fluorenebased copolymers $\mathbf{1 b}$ and $\mathbf{1 d}$, the sidechains of the optimized structures are directed inwards towards the arene units (Fig. 5). This is expected to reduce polymer-polymer backbone interactions leading to higher $\Phi_{\mathrm{PL}}$. For 1a and phenylene-based 1c,

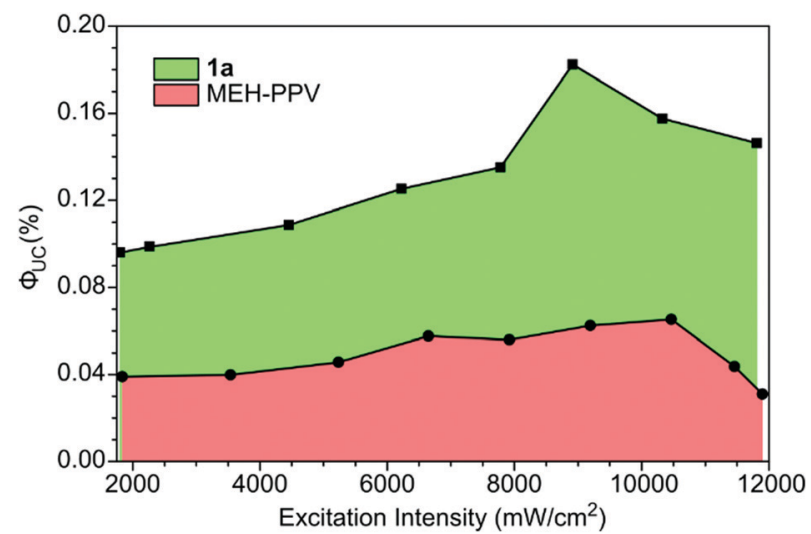

Fig. 4 Dependence of upconversion quantum yield on excitation intensity for PPE 1a $\left(0.25 \mathrm{mg} \mathrm{mL}^{-1}\right), \mathrm{MEH}-\mathrm{PPV}\left(0.5 \mathrm{mg} \mathrm{mL}^{-1}\right)$ with PdTPTBP $(7.5 \mu \mathrm{M})$ as the sensitizer in chloroform solution under $632 \mathrm{~nm}$ excitation. 


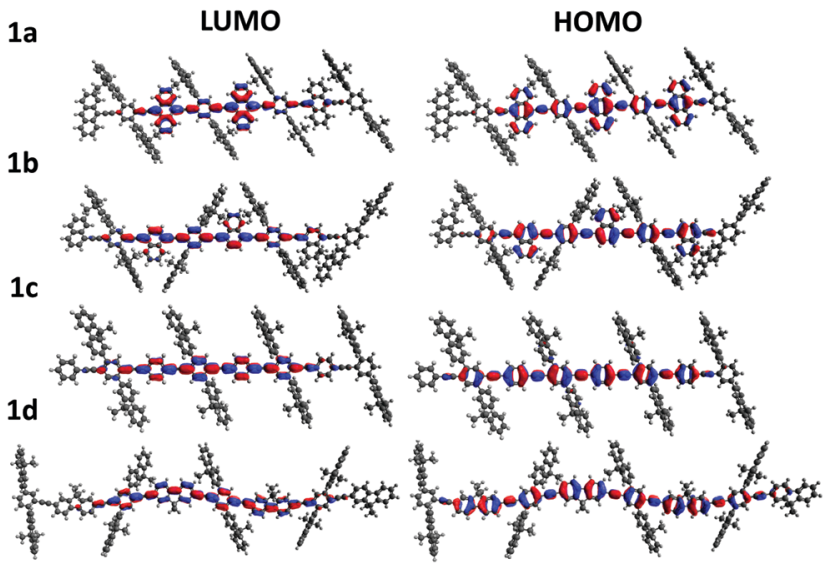

Fig. 5 DFT calculations of model oligomers of $1 \mathbf{a}-\mathbf{d}$ displaying the electron probability distributions for the highest occupied molecular orbitals (HOMO) and lowest unoccupied molecular orbitals (LUMO) for various aryl comonomer units.

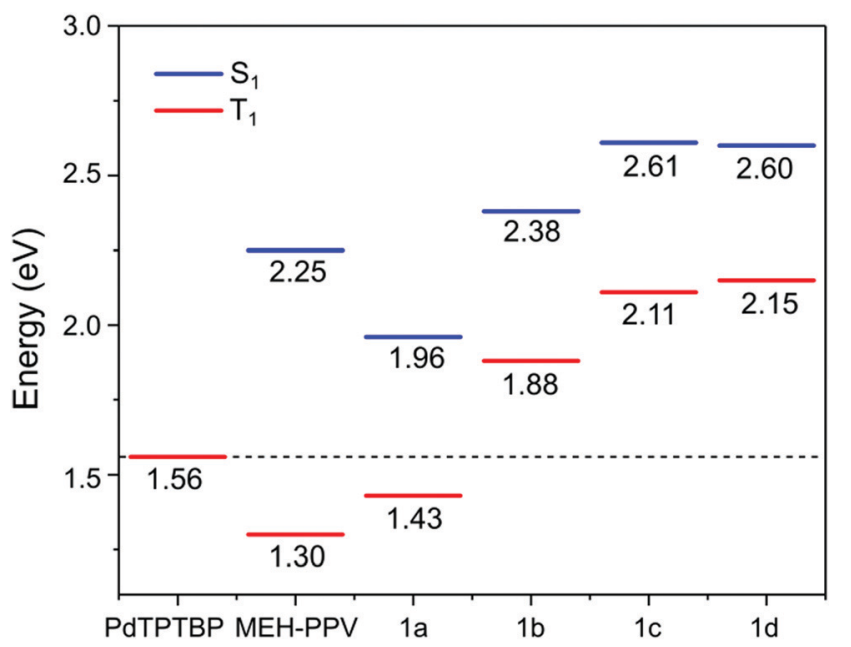

Fig. 6 Energy diagram depicting the calculated singlet and triplet excited state energies obtained by DFT of the model oligomers of $1 \mathbf{a}-\mathbf{d}$ compared to the triplet excited state energy of PdTPTBP. The values for MEH-PPV are taken from ref. 34

the fluorene sidechains occupied the same plane, leaving the polymer backbone more exposed.

The electron distributions for the HOMO and LUMO of all polymers showed a high level of overlap (Fig. 5). Additionally, for the anthracene polymer, more of the electron distribution was found on the anthracene unit itself than for the other polymers, which mainly reside on the conjugated polymer backbone. This may explain the retained vibronic emission features seen in anthracene-based polymer 1a (Fig. 2).

The calculated singlet $\left(\mathrm{S}_{1}\right)$ and triplet $\left(\mathrm{T}_{1}\right)$ energies are shown in Fig. 6 . These values are similar to a previous theoretical study on the energy levels of PPEs by Kohler and Beljonne, where $\mathrm{T}_{1}$ of 1.51, 2.40 and $2.25 \mathrm{eV}$ were found for the anthracene, phenylene and dioctylfluorene-based polymers respectively. ${ }^{33}$ The $T_{1}$ values of $1 \mathrm{a}$ and MEH-PPV, at $1.43 \mathrm{eV}$ and $1.30 \mathrm{eV}^{34}$ respectively, were lower than $\mathrm{T}_{1}$ of the triplet sensitizer PdTPTBP. ${ }^{35}$ This indicated that energetically favourable triplet energy transfer from the sensitizer was possible for 1a and MEH-PPV. This agreed with observations in the phosphorescence quenching and TTA-UC emission measurements.

Although alternative triplet sensitizers could theoretically produce upconversion with copolymers 1b-d, no single sensitizer can work with all polymers in this study given the energy level requirements for TTA-UC. Instead, many of the polymers could be excited directly at sufficiently high excitation energies in preference to a sensitizer, resulting in down-converted emission. As the primary aim of this study was to assess the performance of conjugated polymers as emitters for upconversion and MEH-PPV/PdTPTBP was the reference system, only PdTPTBP was used.

\section{Conclusions}

In summary, a series of PPE copolymers with a variety of aryl spacer units were synthesized and tested as emitters for TTA upconversion. Commercially available conjugated polymers, MEH-PPV and super yellow PPV, were used as references for $\Phi_{\mathrm{UC}}$ measurements after their $\Phi_{\mathrm{UC}}$ values were determined by absolute quantum yield method. The anthracene-based polymer 1a outperformed both references achieving maximum $\Phi_{\mathrm{UC}}$ of $0.18 \%$ with $632 \mathrm{~nm}$ excitation and $8910 \mathrm{~mW} \mathrm{~cm}^{-2}$ intensity. Other copolymers in the series did not quench the phosphorescence of the sensitizer and therefore showed no upconverted emission. DFT calculations indicated only $1 \mathbf{a}$ has the appropriate triplet energy for triplet energy transfer from the PdTPTBP sensitizer. We are in the process of exploring the TTA-UC performance of a wider range of conjugated polymers to further assess their viability in photon upconversion applications.

\section{Abbreviations}

TTA

UC

PPV

PPE

DP

UV-Vis

PL

PdTPTBP

DFT

TD

HOMO

LUMO
Triplet-triplet annihilation

Upconversion

Poly(phenylene-vinylene)

Poly(phenylene-ethynylene)

Degree of polymerization

Ultraviolet-visible

Photoluminescence

Palladium(II)

meso-tetraphenyltetrabenzoporphyrin

Density functional theory

Time dependent

Highest occupied molecular orbital

Lowest occupied molecular orbital

\section{Conflicts of interest}

There are no conflicts to declare. 


\section{Acknowledgements}

This work was supported by the ARC Centre of Excellence in Exciton Science (CE170100026). We thank Prof Kenneth Ghiggino for helpful discussions on photophysical analysis of the upconversion systems.

\section{References}

1 T. F. Schulze and T. W. Schmidt, Energy Environ. Sci., 2015, 8, 103-125.

2 Y. Y. Cheng, B. Fückel, R. W. MacQueen, T. Khoury, R. G. C. R. Clady, T. F. Schulze, N. J. Ekins-Daukes, M. J. Crossley, B. Stannowski, K. Lips and T. W. Schmidt, Energy Environ. Sci., 2012, 5, 6953-6959.

3 W. Shockley and H. J. Queisser, J. Appl. Phys., 1961, 32, 510-519.

4 N. Nishimura, J. R. Allardice, J. Xiao, Q. Gu, V. Gray and A. Rao, Chem. Sci., 2019, 10, 4750-4760.

5 E. M. Gholizadeh, S. K. K. Prasad, Z. L. Teh, T. Ishwara, S. Norman, A. J. Petty, J. H. Cole, S. Cheong, R. D. Tilley, J. E. Anthony, S. Huang and T. W. Schmidt, Nat. Photonics, 2020, 14, 585-590.

6 J. Zhou, Q. Liu, W. Feng, Y. Sun and F. Li, Chem. Rev., 2015, 115, 395-465.

7 C. Gao, S. K. K. Prasad, B. Zhang, M. Dvořák, M. J. Y. Tayebjee, D. R. McCamey, T. W. Schmidt, T. A. Smith and W. W. H. Wong, J. Phys. Chem. C, 2019, 123, 20181-20187.

8 V. Gray, A. Dreos, P. Erhart, B. Albinsson, K. Moth-Poulsen and M. Abrahamsson, Phys. Chem. Chem. Phys., 2017, 19, 10931-10939.

9 T. Singh-Rachford and F. Castellano, J. Phys. Chem. A, 2008, 112, 3550-3556.

10 D. Hertel, H. Bässler, R. Guentner and U. Scherf, J. Chem. Phys., 2001, 115, 10007-10013.

11 F. Laquai, G. Wegner, C. Im, A. Büsing and S. Heun, J. Chem. Phys., 2005, 123, 074902.

12 S. Baluschev, P. E. Keivanidis, G. Wegner, J. Jacob, A. C. Grimsdale, K. Müllen, T. Miteva, A. Yasuda and G. Nelles, Appl. Phys. Lett., 2005, 86, 061904.

13 S. Baluschev, J. Jacob, Y. S. Avlasevich, P. E. Keivanidis, T. Miteva, A. Yasuda, G. Nelles, A. C. Grimsdale, K. Müllen and G. Wegner, ChemPhysChem, 2005, 6, 1250-1253.
14 V. Jankus, E. W. Snedden, D. W. Bright, V. L. Whittle, J. A. G. Williams and A. Monkman, Adv. Funct. Mater., 2013, 23, 384-393.

15 I. Lyskov, E. Trushin, B. Q. Baragiola, T. W. Schmidt, J. H. Cole and S. P. Russo, J. Phys. Chem. C, 2019, 123, 26831-26841.

16 Y. Zhou, F. N. Castellano, T. W. Schmidt and K. Hanson, ACS Energy Lett., 2020, 5, 2322-2326.

17 A. Monguzzi, R. Tubino, S. Hoseinkhani, M. Campione and F. Meinardi, Phys. Chem. Chem. Phys., 2012, 14, 4322-4332.

18 S. M. Bachilo and R. B. Weisman, J. Phys. Chem. A, 2000, 104, 7711-7714.

19 Y. Y. Cheng, T. Khoury, R. G. C. R. Clady, M. J. Y. Tayebjee, N. J. Ekins-Daukes, M. J. Crossley and T. W. Schmidt, Phys. Chem. Chem. Phys., 2010, 12, 66-71.

20 F. Deng, J. Blumhoff and F. N. Castellano, J. Phys. Chem. A, 2013, 117, 4412-4419.

21 T. N. Singh-Rachford and F. N. Castellano, Coord. Chem. Rev., 2010, 254, 2560-2573.

22 N. Yanai, K. Suzuki, T. Ogawa, Y. Sasaki, N. Harada and N. Kimizuka, J. Phys. Chem. A, 2019, 123, 10197-10203.

23 U. H. F. Bunz, Chem. Rev., 2000, 100, 1605-1644.

24 P. Wautelet, M. Moroni, L. Oswald, J. Le Moigne, A. Pham, J. Y. Bigot and S. Luzzati, Macromolecules, 1996, 29, 446-455.

25 J. M. Hong, H. N. Cho, D. Y. Kim and C. Y. Kim, Synth. Met., 1999, 102, 933-934.

26 D. Y. Kim, J. M. Hong, J. K. Kim, H. N. Cho and C. Y. Kim, Macromol. Symp., 1999, 143, 221-230.

27 L. Kloppenburg, D. Jones and U. H. F. Bunz, Macromolecules, 1999, 32, 4194-4203.

28 B.-H. Sohn, K. Kim, D. S. Choi, Y. K. Kim, S. C. Jeoung and J.-I. Jin, Macromolecules, 2002, 35, 2876-2881.

29 A. C. Grimsdale, K. L. Chan, R. E. Martin, P. G. Jokisz and A. B. Holmes, Chem. Rev., 2009, 109, 897-1091.

30 R. O'Shea and W. W. H. Wong, Polym. Chem., 2020, 11, 2831-2837.

31 E. W. Snedden, L. A. Cury, K. N. Bourdakos and A. P. Monkman, Chem. Phys. Lett., 2010, 490, 76-79.

32 T. Ogawa, N. Yanai, A. Monguzzi and N. Kimizuka, Sci. Rep., 2015, 5, 10882.

33 A. Köhler and D. Beljonne, Adv. Funct. Mater., 2004, 14, 11-18. 34 P. C. Jha, E. Jansson and H. Ågren, Chem. Phys. Lett., 2006, 424, 23-27.

35 R. Tao, J. Zhao, F. Zhong, C. Zhang, W. Yang and K. Xu, Chem. Commun., 2015, 51, 12403-12406. 